\title{
Pharmacological management of low milk supply with domperidone: separating fact from fiction
}

\section{Restrictions require scrutiny, as their relevance to young and otherwise healthy lactating women is uncertain}

xclusive breastfeeding for the first 6 months of life is well recognised as the optimal form of nutrition to support the growth and development of term and preterm infants. ${ }^{1}$ Whether real or just perceived, low milk supply is the most commonly reported reason for early discontinuation of breastfeeding. ${ }^{2}$ This is despite the fact that most mothers are able to produce sufficient quantities of milk for their baby, if given the correct support and information. ${ }^{3}$ Risk factors for women experiencing low milk supply include maternal illness, caesarean birth, maternal smoking, previous breast surgery, motherinfant separation and indirect lactation (ie, breast pump or manual milk expression). ${ }^{3}$ Mothers of infants who are unwell and require supportive care after birth, such as preterm infants, are particularly vulnerable to experiencing low milk supply. ${ }^{3}$

In many of these situations, non-pharmacological strategies such as optimising infant positioning and attachment at the breast, increasing the frequency of breastfeeds and mechanical expression are usually sufficient to increase a woman's milk supply. ${ }^{3}$ However, some women still struggle to establish or maintain an adequate milk supply. In these situations, pharmacological intervention with medications used to augment lactation, known as galactagogues, is warranted. The most widely studied and commonly used galactagogues are the dopamine receptor antagonists, metoclopramide and domperidone. Both medications stimulate prolactin release by blocking dopamine D2 receptors in the anterior pituitary. Although studies are still few, these drugs have been shown to be effective treatments for the initiation and maintenance of lactation. ${ }^{4,5}$ In recent years, domperidone has become increasingly used in preference to metoclopramide, as it does not cross the blood-brain barrier, is associated with fewer central nervous system side effects (ie, fatigue, irritability, depression), and is secreted in only small amounts into breast milk. ${ }^{4}$

Recently, albeit not for the first time, warnings surrounding the use of domperidone and the potential for adverse cardiac effects have arisen. ${ }^{6}$ However, these warnings require greater scrutiny, as their relevance to young and otherwise healthy lactating women is uncertain. ${ }^{5}$ The QT-prolonging effects of domperidone, which place susceptible users at a significantly increased risk of fatal cardiac arrhythmias, have been well known for years. ${ }^{6}$ Of particular interest is the concept of susceptibility, as the early case reports linking domperidone use to sudden cardiac death describe the administration of high-dose intravenous formulations in patients with cancer who also had significant electrolyte disturbances. These reports led to the worldwide withdrawal of the intravenous formulation in $1985 .{ }^{6}$ The oral form has remained available for use in many countries, including Australia, although it has never received marketing approval in the United States.

Additional evidence supporting the association between the use of domperidone and sudden cardiac death emerged through the publication of two casecontrol studies in 2010.,8 These additional concerns led to further regulatory warnings and ultimately prompted a review of all domperidone-containing medicines by the Pharmacovigilance Risk Assessment Committee (PRAC) of the European Medicines Agency (EMA) in 2013. The findings of this review have recently been released. The committee determined that benefits in relation to the symptomatic management of nausea and vomiting outweighed associated risks, although they recommended that the daily oral dose be restricted to a maximum of $30 \mathrm{mg}$ and that domperidone not be used for longer than 1 week. ${ }^{6}$

doi: 10.5694/mjal4.00626 But what about its use among lactating women? An important caveat of this review is that it sought to determine whether the benefits of domperidone use outweighed associated risks only for its approved indications. Given that the use of domperidone as a galactagogue does not reflect an approved indication, it was therefore not considered within this investigation. This could reflect a significant oversight, given domperidone is commonly used off-label for this indication. It also raises significant questions regarding the generalisability of previous study findings on which these recommendations were based, and therefore the relevance of this EMA PRAC recommendation. Issues regarding generalisability are highlighted through findings from one of the two most recent studies, where an increased risk of sudden cardiac death associated with domperidone use was only observed among subjects $>60$ years of age (adjusted odds ratio [aOR], 1.64; 95\% CI, 1.31-2.05), and not among those $\leqslant 60$ years of age (aOR, $1.1 ; 95 \%$ CI, 0.35-3.47). ${ }^{7}$ Furthermore, an increased risk was only observed among males (aOR, 2.23; 95\% CI, 1.59-3.13), but not females (aOR, 1.25; 95\% CI, 0.93-1.67) of any age. ${ }^{7}$ Therefore, one could expect that any increased risk associated with being female and $\leqslant 60$ years would be negligible.

Where does this leave clinical practice? An Australian-based clinical audit from 2000 to 2010 showed that domperidone is commonly used off-label 
as a galactagogue in clinical practice, and that by $2010,5 \%$ of all women giving birth at a major tertiary teaching hospital received domperidone. ${ }^{9}$ It is likely, however, that this reflects an underestimation of total use, as medication data were obtained from the hospital pharmacy alone and domperidone may also be prescribed in community settings, for which no data are currently available. Despite its common use in many settings around Australia and overseas, no case reports of sudden cardiac death or serious ventricular arrhythmias associated with its use in lactating women have emerged. This could reflect the rarity of this outcome among women of childbearing age, so we may never be able to generate a clear answer on the associated risks in this population, as the total number of lactating women needed in studies would be very large. However, with a reported incidence for cardiac-related out-of-hospital cardiac arrests among young adults aged 16-39 years of 6 per $100000,{ }^{10}$ the absolute risks associated with the use of domperidone as a galactagogue are likely to be very small, if significant at all. Furthermore, a perspective not often considered in previous discussions is the infant and maternal benefits offered by breastfeeding. This perspective is essential, as breastfeeding is associated with significant reductions in infant morbidity and mortality, ${ }^{1,2}$ as well as providing longterm benefits for the mother, with reductions in the incidence of certain cancers, for example. ${ }^{2}$ It could be considered that these wide-ranging benefits greatly outweigh what amounts to, at this point in time, largely theoretical risk associated with the use of domperidone as a galactagogue. ${ }^{5}$

While research to determine the optimal treatment regimens for domperidone is ongoing, five clinical recommendations can be drawn from existing evidence to guide its safe and effective use.

- Before considering domperidone, ensure that low milk supply persists despite the adequate trial of non-pharmacological approaches. This is a clinical decision that may require input from a lactation consultant. While ongoing studies seek to identify the most appropriate time to initiate treatment with domperidone, low milk supply may be identified as early as 7 days postpartum.

- Ensure mothers are appropriately screened for any comorbid medical conditions (including a significant personal or family history of cardiac arrhythmia), concurrent use of other medications that may prolong the QT interval and/or inhibit the metabolism of domperidone (eg, macrolide antibiotics and triazole antifungals).

- Appropriately discuss the benefits and risks of the use of domperidone to increase breast milk supply to ensure mothers make an informed decision.

- Prescribe domperidone at an initial dose of $10 \mathrm{mg}$ three times daily. A response to treatment should be evident within 7 days, with maximal effects likely to be achieved after 2 to 4 weeks. ${ }^{5}$ If supply remains low, increasing the dose to $20 \mathrm{mg}$ three times daily may be beneficial. No clinical studies have investigated the effects of doses greater than $20 \mathrm{mg}$ three times daily. Once an adequate breast milk supply is achieved, women may benefit from titrating the dose downwards over 1 to 2 weeks before ceasing, avoiding an abrupt withdrawal of treatment.

- Ensure regular follow-up to confirm adherence to non-pharmacological management approaches and monitor closely for improvements in milk supply and the presence of any side effects associated with domperidone.

Used in accordance with these clinical recommendations, it is possible that domperidone may be used without direct risk to breastfeeding women. At least two major studies are already underway that will help further guide the safe and effective use of domperidone. These studies will improve our understanding of the most effective time to initiate domperidone treatment postpartum and the optimal dose and duration of treatment, and they will explore why some women do and do not respond to treatment with domperidone, which may allow further medication tailoring.

In conclusion, domperidone, or the use of any other galactagogues for that matter, should never be considered a magic bullet. They should not be used as a substitute for, or as an alternative to, effective non-pharmacological breastfeeding support and encouragement; but domperidone can be used safely and effectively. As for further regulatory action, it is essential that benefits and risks be judged objectively, regardless of its off-label status. The provision of further restrictions to the use of domperidone may do more harm than good, with the risk of condemning lactating women to the status of therapeutic orphans.

Acknowledgement: Luke Grzeskowiak acknowledges salary support from a National Health and Medical Research Council Australian Public Health Fellowship (1070421).

Competing interests: No relevant disclosures

Provenance: Not commissioned; externally peer reviewed.

1 Stuebe AM, Schwarz EB. The risks and benefits of infant feeding practices for women and their children. $J$ Perinatol 2010; 30: 155-162.

2 Odom EC, Li R, Scanlon KS, et al. Reasons for earlier than desired cessation of breastfeeding. Pediatrics 2013; 131: e726-e732.

3 Amir LH. Breastfeeding: managing 'supply' difficulties. Aust Fam Physician 2006; 35: 686-689.

4 Zuppa AA, Sindico P, Orchi C, et al. Safety and efficacy of galactogogues: substances that induce, maintain and increase breast milk production.

J Pharm Pharm Sci 2010; 13: 162-174.

5 Osadchy A, Moretti ME, Koren G. Effect of domperidone on insufficient lactation in puerperal women: a systematic review and meta-analysis of randomized controlled trials. Obstet Gynecol Int 2012; 2012: 642893.

6 European Medicines Agency. PRAC recommends restricting use of domperidone. London, UK: EMA, 2014 http://www.ema.europa.eu/docs/en_GB/document_library/Referrals_document/Domperidone_31/ Recommendation_provided_by_Pharmacovigilance_Risk_Assessment_Committee/WC500162559.pdf (accessed Apr 2014).

7 Johannes CB, Varas-Lorenzo C, McQuay LJ, et al. Risk of serious ventricular arrhythmia and sudden cardiac death in a cohort of users of domperidone: a nested case-control study. Pharmacoepidemiol Drug Saf 2010; 19: 881-888.

8 van Noord C, Dieleman JP, van Herpen G, et al. Domperidone and ventricular arrhythmia or sudden cardiac death: a population-based case-control study in the Netherlands. Drug Saf 2010; 33: 1003-1014.

9 Grzeskowiak LE, Lim SW, Thomas AE, et al. Audit of domperidone use as a galactogogue at an Australian tertiary teaching hospital. J Hum Lact 2013; 29: 32-37.

10 Deasy C, Bray JE, Smith K, et al. Out-of-hospital cardiac arrests in young adults in Melbourne, Australia. Resuscitation 2011; 82: 830-834. 